\title{
Correlation between body mass index and maximum bite force in young adults
}

\author{
Silvana Bommarito*, Patricia Barbarini Takaki, Angelica da Veiga Said, Luciana Escanoela Zanato and Marilena Manno Vieira \\ The Federal University of São Paulo, Brazil
}

\begin{abstract}
Maximum bite force $(\mathrm{MBF})$ is considered as the result of the maturation of the stomatognathic system and its actual functional condition, variations, and adaptations, as well as the chewing preferences of individuals. It is closely related to dental occlusion and craniofacial characteristics.

Objective: To determine whether a correlation exists between body mass index (BMI) and MBF in young adults without occlusal and orofacial myofunctional changes.

Methods: The cohort included 124 individuals from the city of São Paulo, aged between 18 and 45 years (mean, 22 years), who had complete permanent dentition and normal occlusion or Angle Class I malocclusion (1899) and were selected independently according to BMI. The study was approved by Ethics Committee of UNIFESP/HSP (No. 288,430). The individuals were evaluated by an orthodontist, who checked their dental conditions and classified their occlusions. The participants underwent a brief medical history taking, and BMI and MBF assessments. MBF was measured in Newtons by using a digital dynamometer model DDK/M (Kratos, São Paulo, Brazil). Patients with obesity were identified.

Results: The analysis results showed that the number of individuals with normal BMI was equal to that of obese individuals among the men. The proportion of obese women was $32 \%$ lower than that of obese men. Moreover, more women than men had BMIs within the normal range, while a higher number of men were found to have BMIs within the obesity range. BMI correlated with the mean MBF on the right and left sides and total mean MBF. No statistically significant correlations were found between the variables ( $p>0.05$ ); we only noted a poor correlation between the variables assessed.
\end{abstract}

Conclusion: High BMI values, despite being an indicator of impaired muscle functions, could not provide a significant assessment of masseter muscle function, as no correlation with MBF was observed.

\section{Introduction}

Maximum bite force (MBF) is an objective chewing measure that indicates muscle efficiency [1], dental occlusion, and development of masticatory function [1-3], and takes into account the conditions of the stomatognathic system [2].

Several ways have been developed to evaluate chewing. However, after years of research, MBF has been inferred to be an important parameter to assess maturation of the stomatognathic system [3] and its actual functional condition, changes, and adaptations, as well as the chewing preferences of individuals throughout the period in which they learn masticatory movements [4]. Moreover, it directly takes into account dental occlusion $[1,5,6]$ and craniofacial characteristics [6,7].

International literatures report the existence of correlations between bite force and body mass index (BMI) [6] and obesity [8], showing that bite force influences the muscle strength of the entire body and is due to an endocrine reaction that leads to the release of adipokines and inflammatory markers. This results in high levels of free fatty acids accumulating in skeletal muscles $[9,10]$ and reduced muscular strength in individuals with obesity, when compared with subjects who were never obese [10].

In clinical practice, a difference in muscle strength of the stomatognathic system was observed between individuals with different BMIs. However, clinicians should bear in mind that individuals with high BMIs could present more-flaccid tissues and take longer time to attain the muscle tone needed for masticatory function and speech.
However, no consensus has been reached regarding the correlation between BMI and bite force, as a strong relationship with obesity has been found in one study [8], a weak relationship in two studies [1,6], and no relationship in other studies $[3,4,7]$. Furthermore, only few studies have investigated the association between BMI and bite force. Therefore, more investigations are required to understand the effects of $\mathrm{BMI}$ on muscles function, especially on bite force.

Considering the importance of this subject in speech therapy in clinical practice and other areas of health, particularly in assessments, prognosis, therapy, and the importance of understanding the effects of BMI on mastication, this study aimed to investigate whether a correlation exists between BMI and MBF in young adults without occlusal and orofacial or myofunctional changes.

\section{Methods}

This study was approved by the research ethics committee of Federal University of São Paulo (UNIFESP)/HSP (No. 288,430), and its development was financially supported by the Coordination for the Improvement of Higher Education Personnel (CAPES). All volunteers agreed and signed the informed consent form.

Correspondence to: Silvana Bommarito, Rua Botucatu, 802 - Vila Clementino CEP: 04023-900 São Paulo - SP/ Brazil, E-mail: bomarito@terra.com.br

Key words: bite force, body mass index, occlusion

Received: July 22, 2016; Accepted: August 10, 2016; Published: August 15, 2016 
The cohort included 124 individuals from the city of São Paulo, aged between 18 and 45 years (mean, 22 years), who had complete permanent dentition and normal occlusion or Angle class I malocclusion [11] (1899), and were randomly selected, that is, regardless of BMI.

Individuals with smoking habits and/or alcoholism, syndromes and birth defects, mental and physical disabilities, and previous cranioencephalic trauma or head and neck surgery were not included in the study.

Individuals with nasal obstruction, orofacial myofunctional alterations, open or cross bite, dental malocclusions (except Angle class I, 1899), and temporomandibular disorders (TMD) were excluded from the study. Individuals under orthodontic treatment or who had been treated for $<2$ years, as well as individuals with implants or dental mutilations were also excluded.

\section{Experiment}

All procedures were performed in the Orofacial Motricity Outpatient Clinic of the UNIFESP, located in the city of São Paulo. All volunteers underwent an orthodontic evaluation by an orthodontist, who assessed dental conditions and classified the occlusion, a step in the sample selection. All the subjects consistently received accurate and detailed instructions for all procedures and evaluations.

For biosecurity reasons, all instruments used for the evaluations were cleaned with $70 \%$ alcohol, and the bite rod of the equipment used to measure MBF was secured with disposable latex finger cots, always after each procedure. All measurements were performed three times to increase the reliability of the data, and the average of the three measurements was then calculated.

\section{Anamnesis}

Anamnesis was performed to obtain personal identification data.

\section{Evaluation of the BMI}

The body mass index (BMI) was calculated by the weight coefficient divided by the height squared, as formulated by Quetelet [12]. Weight was measured in kilograms by using a digital scale, on which each individual was positioned with the back straight, the head in the Frankfurt horizontal plane, parallel feet, and bare feet, dressed with light clothing and standing in the middle of the equipment platform. In the same position, the subjects' heights were measured with a stadiometer (Table 1).

\section{Evaluation of the MBF}

Evaluation of MBF was performed by using a digital dynamometer model DDK/M (Kratos, São Paulo, Brazil). This device was designed to determine the force applied by an individual at the time of the bite (capacity of up to $100 \mathrm{Kgf}$, adapted to oral conditions). This was measured by means of the Newton scale $(\mathrm{N})$ and recorded as the peak of the force exerted during the biting. The value was then shown on a digital display.

Table 1. Body Mass Index Classification [12]

\begin{tabular}{|c|c|}
\hline Degree of obesity & BMI value $\left(\mathrm{kg} / \mathrm{m}^{2}\right)$ \\
\hline Normality & $20,0-25,0$ \\
\hline Obesity class I & $25,1-29,9$ \\
\hline Obesity class II & $30,0-34,9$ \\
\hline Obesity class III & $35,0-39,9$ \\
\hline Obesity class IV & $\geq 40$ \\
\hline
\end{tabular}

The participants were seated with their backs straight and heads in the Frankfurt horizontal plane, on the median sagittal plane. The device was placed in the region of the first molars of each evaluated individual, and the participant was instructed to bite it as strong as possible. Then, three records on each side, with an interval of 2 minutes between them, were performed in an alternate manner. The mean of the three measurements was subsequently calculated.

\section{Data analysis}

Data were statistically analyzed by using parametric tests, and a significance level of 0.05 (5\%) and statistical confidence of $95 \%$ were adopted for the results obtained in this study. $\mathrm{P}$ values were also calculated.

The Pearson correlation test was used to measure how the variables were interrelated. The results were expressed as percentages, which could be positive or negative. Positive values were obtained when the relationship between the variables assessed was proportionately positive, whereas the contrary indicates that the relationship was inversely correlated. The grading scale below determines the relative intensity level (Figure 1).

\section{Results}

The study sample was divided according to sex, and the distribution of individuals with normal or obese BMIs was analyzed. The following distributions according to BMI and sex were observed: By grouping according to obese classifications, we found that in men, the number of individuals with normal BMI was equal to that of obese individuals. Meanwhile, the number of obese women was 32\% lower than the number of obese men, as shown in Table 2.

In order to analyze the relationship between $\mathrm{BMI}$ and MBF, BMI was correlated to the mean MBF values of the right and left sides, and to the total mean MBF. Table 3 shows no statistically significant relationship between the variables analyzed $(p>0.05)$. However, a weak

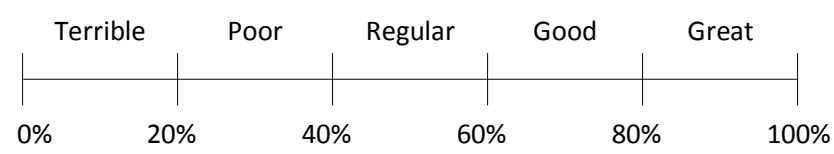

Figure 1. The results were expressed as percentages, which could be positive or negative.

Table 2. Sample distribution by sex and BMI class.

\begin{tabular}{|c|c|c|c|c|c|c|c|c|}
\hline & Sex & & $\mathrm{Ma}$ & & & Fema & & Total \\
\hline BMI & & $\mathbf{N}$ & $\%$ & Mean & $\mathbf{N}$ & $\%$ & Mean & \\
\hline Normal & & 34 & $27 \%$ & 22.16 & 38 & $31 \%$ & 21.47 & 72 \\
\hline Obese & Class I & 26 & $21 \%$ & 27.29 & 14 & $11 \%$ & 27.10 & 40 \\
\hline & Class II & 7 & $6 \%$ & 32.18 & 4 & $3 \%$ & 31.91 & 11 \\
\hline & Class III & 1 & $1 \%$ & 37.72 & 0 & $0 \%$ & 0.00 & 1 \\
\hline & Class IV & 0 & $0 \%$ & 0.00 & 0 & $0 \%$ & 0.00 & 0 \\
\hline & TOTAL & 68 & $55 \%$ & 23.87 & 56 & $45 \%$ & 16.10 & 124 \\
\hline
\end{tabular}

Table 3. Correlation between BMI and the right, left, and total MBF, according to sex.

\begin{tabular}{|c|c|c|c|c|c|c|}
\hline \multirow{2}{*}{ Sex } & \multicolumn{5}{|c|}{ Male } & \multicolumn{3}{c|}{ Female } \\
\cline { 2 - 7 } MBF & \multicolumn{5}{|c|}{ BMI } \\
\cline { 2 - 7 } & Mean & Corr (r) & P-valuer & Mean & Corr (r) & P-value \\
\hline Right & 621,0 & $17,6 \%$ & 0,151 & 384,5 & $0,7 \%$ & 0,961 \\
\hline Left & 617,8 & $14,1 \%$ & 0,251 & 414,5 & $9,9 \%$ & 0,467 \\
\hline Total & 619,4 & $16,3 \%$ & 0,183 & 399,5 & $5,7 \%$ & 0,679 \\
\hline
\end{tabular}

Corr = Correlation; Tendency to statistical significance; Statistically significant correlation; Significant Correlation 
correlation was observed between all the variables.

\section{Discussion}

Although many studies have been published in the literature on $\mathrm{BMI}$, we found that its relationship with MBF still requires elucidation, as investigations on this subject are scarce and far from being recent. Thus, a better understanding on the topic is necessary, as BMI could influence muscle function, this being the object of therapeutic investigations in several health areas, including speech therapy.

In this study, we conducted a random sampling and observed that the number of obese women was $32 \%$ lower than the number of obese men. This was also observed in a Turkish study by Koç et al. [7], in a Chinese study by Sun et al. [8], and in a Brazilian study by Cunha et al. (2015) [13]. Conversely, the study by Dyck et al. (2015) [14] in 10 different countries showed no significant differences between sexes and BMIs. Other studies did not report any findings on this regard.

The fact that Dyck et al. [14] did not find any differences between sexes and BMIs in their study population may be due to the small cohort used to represent the populations of the many countries analyzed. Another possible reason relies on the fact that more obese men than women were included in the study owing to regional characteristics, which are found only in larger and specific investigations conducted in certain countries such as Brazil and China. In the United States, for example, Flegal et al. [15] found no significant difference in the number of obese individuals between sexes, showing that almost one third of the sample were classified as obese.

In relation to MBF, we observed a significant difference between sexes, in agreement with previous studies [1,2,7], being $36 \%$ higher in men than in women. This finding corroborates those found in the literature that reported a difference of $30 \%$. No significant differences in $\mathrm{MBF}$ were found between the two sides, although significant differences were found in a previous study according to masticatory preference [4]. In this investigation, masticatory preference was not analyzed, as it is not within the scope of our study objective.

Studies on the relationship BMI and muscle strength suggest that two variables are not related [16] and that muscle strength is directly related to lean body mass [17]. However, another study reported that individuals who were never obese had greater muscle strength than individuals who had experienced obesity during their lives [10]. This led to the hypothesis that a relationship exists between muscle strength and BMI.

The literature shows that the adipose tissue is not only an energy reserve but interferes with muscle strength as a consequence of an endocrine reaction. The adipose tissue secretes hormones and proinflammatory proteins that disrupt inflammatory response, which are also generated during catabolism and raise the levels of free fatty acids accumulated in skeletal muscles. This increase contributes to the reduction of muscle mass and strength $[9,10]$, thus being then capable to interfere in many functions such as chewing.

MBF is just one way of evaluating chewing. MBF properly translates the integrity of the stomatognathic system and its ability to carry out its functions into an objective measure. In studies that specifically analyzed $\mathrm{MBF}$, the results obtained showed the correlation of weight and height [6] to BMI in adolescents [8]. However, another study that assessed BMI and MBF in adults did not find any relationships between the variables [7]. Our study contributes to reaching a consensus in the literature, as we did not observe a relationship between $\mathrm{BMI}$ and $\mathrm{MBF}$, as shown in Table 3, which is in agreement with the study of Koç et al. [7]. One explanation for this finding not agreeing with the studies of Braun et al. [6] and Sun et al. [8] is that Braun et al. analyzed weight and height separately, while Sun et al. included individuals between 13 and 16 years of age as cohort for their study, which are both notably different methods from that used in this work.

Another possible explanation for BMI not influencing MBF is related to the smaller size of the masseter muscle when compared with the sizes of the other muscle groups such as quadriceps [18], biceps [19], and other study muscles $[10,17,19]$. The literature shows that an important relationship exists between muscle size and force, as muscle volume is the product of the physiological cross-sectional area, which determines the number of sarcomeres that contract, simultaneously generating maximum power, and the length of the fascicle [19]. Therefore, any loss of muscle mass or volume reduction impairs the force exerted by the muscle $[18,20]$, mainly in the larger anterior portion of the superficial head of the masseter muscle [21]. As the muscle volume increases, so does muscle strength [19]. This fact may explain the results of the present study.

An important observation is that muscle strength varies much more than muscle volume, as shown by the following studies: In the sarcopenia studies, while the loss of muscle mass ranged from $0.64 \%$ to $0.70 \%$ per year in women and from $0.80 \%$ to $0.98 \%$ in men, the loss of muscle strength varied from $3 \%$ to $4 \%$ per year in women and from $2.5 \%$ to $3 \%$ in men [20]. On the other hand, a study on resistance training showed that an increase of $10 \mathrm{~cm}^{2}$ in muscle volume increased the muscle strength by $100 \%$ [19].

Thus, as the relationship of BMI to MBF was not significant and therefore negligible, BMI most likely does not influence MBF, having as plausible explanation the smaller muscle size when compared with the sizes of other muscle groups. However, more specific studies are required that equalize populations within BMI classification groups, unravel the possible relationships in grade I, II, III, and IV obesity, and even evaluate the thickness of the masseter muscle by using ultrasonography in these individuals. Only then will it be possible to affirm that BMI does not influence MBF.

During this study, a major difficulty was finding literatures that specifically address the relationship between the masseter muscle and BMI, thus bringing to discussion studies on the impact of obesity on other body muscles. Despite this difficulty, this study contributed to the knowledge about the endocrine effects of adipose tissue and the resulting reaction, and to the better understanding of the relationship between muscle volume and strength.

Studies in this area are important to better understand all the factors that interfere in muscle performance so that they can be recognized and considered in speech therapy clinical practice.

\section{Conclusion}

We can conclude that despite that high BMI values indicate impaired functions of other body muscles, body mass do not significantly influence the functioning of the masseter muscle, as no correlation was found with MBF.

\section{References}

1. Varga S, Spalj S, Lapter Varga M, Anic Milosevic S, Mestrovic S, et al. (2011) Maximum voluntary molar bite force in subjects with normal occlusion. Eur J Orthod 33: 427-433. [Crossref]

2. Palinkas M, Nassar MS, Cecílio FA, Siéssere S, Semprini M, et al. (2010) Age and 
gender influence on maximal bite force and masticatory muscles thickness. Arch Oral Biol 55: 797-802. [Crossref]

3. Takaki P, Vieira M, Bommarito S (2014) Maximum bite force analysis in different age groups. Int Arch Otorhinolaryngol 18: 272-276. [Crossref]

4. Bommarito S, Takaki P, Vieira M (2014) Análise da força máxima da mordida segundo a preferência mastigatória. Rev Clín Ortod Dental Press 13: 91-97.

5. Bakke M (2006) Bite force and occlusion. Semin Orthod 12: 120-126.

6. Braun S, Bantleon HP, Hnat WP, Freudenthaler JW, Marcotte MR, et al. (1995) A study of bite force, part 1: relationship to various physical characteristics. Angle Orthod 65: 367-372.

7. Koç D, Doğan A, Bek B (2011) Effect of gender, facial dimensions, body mass index and type of functional occlusion on bite force. J Appl Oral Sci 19: 274-279. [Crossref]

8. Sun K-T et al. (2015) Bite-force difference among obese adolescents in central Taiwan. J Formosan Med Assoc 1-7.

9. Eckardt K, Taube A, Eckel J (2011) Obesity-associated insulin resistance in skeleta muscle: Role of lipid accumulation and physical inactivity. Rev Endocr Metab Disord 12: $163-172$.

10. Stenholm S, Sallinen J, Koster A, Rantanen T, Sainio P, et al. (2011) Association between obesity history and hand grip strength in older adults-Exploring the roles of inflammation and insulin resistance as mediating factors. J Gerontol A Biol Sci Med Sci 66A: 341-348. [Crossref]

11. Angle EH (1899) Classification of malocclusion. Dental Cosmos 41: 248-264, 350-357.

12. Quetelet A (1991) Sur l'homme et le développementde ses facultés. Paris: Fayard. 1a ed 1835. Paris: Fayard.

13. Cruz MAF, Guimarães MKH, Macena LB, Silva, LNS, et al. (2015) Nível de atividade física e índice de massa corpórea em acadêmicos de medicina da Universidade Tiradentes em Aracaju-SE. Ciências Biológicas e de Saúde 3: 101-112.

14. Van Dyck D, Cerin E, De Bourdeaudhuij I, Hinckson E, Reis RS5, et al. (2015) International study of objectively measured physical activity and sedentary time with body mass index and obesity: IPEN adult study. Int J Obes (Lond) 39: 199-207. [Crossref]

15. Flegal KM, Carroll MD, Kit BK, Ogden CL (2012) Prevalence of obesity and trend in the distribution of body mass index among US adults, 1999-2010. JAMA 307: 491497. [Crossref]

16. Rantanen T, Harris T, Leveille SG, Visser M, Foley D, et al. (2000) Muscle strength and body mass index as long-term predictors of mortality in initially healthy men. $J$ Gerontol A Biol Sci Med Sci 55: M168-173. [Crossref]

17. Barbat-Artigas S, Plouffe S, Pion CH, Aubertin-Leheudre M (2013) Toward a sexspecific relationship between muscle strength and appendicular lean body mass index? J Cachexia Sarcopenia Muscle 4: 137-44.

18. Young A, Stokes M, Crowe M (1984) Size and strength of the quadriceps muscles of old and young women. Eur J Clin Invest 14: 282-287. [Crossref]

19. Mitchell WK, Williams J, Atherton P, Larvin M, Lund J, et al. (2012) Sarcopenia, dynapenia, and the impact of advancing age on human skeletal muscle size and strength; a quantitative review. Front Physiol 3: 260. [Crossref]

20. Hubal MJ, Gordish-Dressman H, Thompson PD, Price TB, Hoffman EP, et al. (2005) Variability in muscle size and strength gain after unilateral resistance training. Med Sci Sports Exercise 1: $964-972$.

21. Bakke M, Tuxen A, Vilmann P, Jensen BR, Vilmann A, et al. (1992) Ultrasound image of human masseter muscle related to bite force, electromyography, facial morphology, and occlusal factors. Scand J Dent Res 100: 164-171. [Crossref]

Copyright: $\left({ }^{2} 2016\right.$ Takaki PB. This is an open-access article distributed under the terms of the Creative Commons Attribution License, which permits unrestricted use, distribution, and reproduction in any medium, provided the original author and source are credited. 